\title{
Cardiac tissue characterization using near-infrared spectroscopy
}

\author{
Rajinder Singh Moon and Christine P. Hendon \\ Department of Electrical Engineering. Columba University. New York, NY.
}

\begin{abstract}
Cardiac tissue from swine and canine hearts were assessed using diffuse reflectance near-infrared spectroscopy (NIRS) ex vivo. Slope measured between $800-880 \mathrm{~nm}$ reflectance was found to reveal differences between epicardial fat and normal myocardium tissue. This parameter was observed to increase monotonically from measurements obtained from the onset of radiofrequency ablation (RFA). A sheathe-style fiber optic catheter was then developed to allow real-time sampling of the zone of resistive heating during RFA treatment. A model was developed and used to extract changes in tissue absorption and reduced scattering based on the steady-state diffusion approximation. It was found that key changes in tissue optical properties occur during application of RF energy and can be monitored using NIRS. These results encourage the development of NIRS integrated catheters for real-time guidance of the cardiac ablation treatment.
\end{abstract}

Keywords: Diffuse reflectance, near-infrared spectroscopy, radiofrequency ablation, arrhythmia, Monte Carlo simulation, diffusion approximation

*rs3226@columbia.edu ,1-212-854-2280

\section{BACKGROUND}

Cardiac arrhythmias afflict millions of patients in the United States, resulting in frequent hospitalizations and high costs for hospitalizations and medications[1]. Medications and devices do not cure arrhythmias. Currently the only curative treatment for arrhythmias is radiofrequency ablation (RFA), and RFA is the standard of care for treatment of many arrhythmias. RFA destroys tissue that triggers abnormal electrical pathways[2], by creating discrete areas of necrosis on the endocardial surface of the heart which interrupt electrical conduction. Since pharmacological therapies have limited effectiveness, catheter ablation directed at interrupting critical components of arrhythmia circuits has emerged as a prominent approach for the treatment of a broad range of atrial and ventricular tachyarrhythmias.

The goal of RFA is to target and destroys tissue that triggers or supports abnormal electrical pathways, while minimizing or avoiding damage to normal areas of the heart. The monitoring of successful formation of an ablation lesion is only by indirect means by measuring temperature and impedance of the surface of the electrode-tissue interface. This limited, indirect method of monitoring during ablation procedures often results in delivering more ablation lesions than necessary to achieve the therapeutic effect, prolonging procedure times, thereby limiting the effectiveness and increasing risk of this procedure. We propose to use optical spectroscopy as a means to monitor RFA treatment of cardiac arrhythmias, which will directly interrogate the tissue for characterization regardless of the type of ablation catheter used.

At present, the duration of RFA procedures range from 3 to 12 hours. Moreover, some RFA procedures to treat atrial fibrillation are associated with the delivery of dozens of lesions[3] producing injury to normal myocardial muscle. Guidance is necessary to reduce the number of complications associated with RFA treatment. Within the 2002 report by the FDA, $95 \%$ of ablation procedures are acutely successful, $90 \%$ are chronically successful and $2.5 \%$ have major complications[4]. The complications associated with RFA vary depending on arrhythmia targeted. Complex ablations such as ventricular tachycardia or atrial tachycardia may have complication rates up to $8 \%$. A global survey of 777 institutions, and 8745 RFA procedures for the treatment of atrial fibrillation showed a $76 \%$ chronic success and $6 \%$ complication rate [5]. However, when considering procedural success in the absence of anti-arrythmia drugs, there was a

Photonic Therapeutics and Diagnostics X, edited by Bernard Choi, et al., Proc. of SPIE Vol. 8926, 89263N - (C) 2014 SPIE - CCC code: 1605-7422/14/\$18 - doi: 10.1117/12.2036320 
$52 \%$ chronic success, and the other $24 \%$ required medication to be chronically arrhythmia free. It was also noted that medical centers that conduct more RFA procedures for the treatment of AF have higher chronic success rates[5].

Importantly, the long-term complications associated with delivery of numerous RF lesions are not well reported or studied. Cardiac perforation is one of the most serious complications related to RFA therapy, with a 0.1 to $0.7 \%$ incidence[2]. Cardiac perforations have been shown to be associated with high impedance rise. An optical-based monitoring system capable of assessing tissue contact, validating lesion formation, and discerning early signs of overtreatment may help to increase efficacy and reduce complication rates.

Fluoroscopy, low dosage, real-time X-ray, is the standard imaging tool used to guide RFA therapy. Fluoroscopy is used to navigate the ablation catheter to specific areas within the heart chambers and assess cathetertissue contact. Magnetic resonance imaging (MRI) $[6,7]$ and CT $[8,9]$ have been used to obtain the three dimensional anatomy of the heart for procedure planning and has been recently used for post procedural evaluation. Structural information provided by these modalities aid in interpreting electrograms and 3D voltage maps. Other than fluoroscopy, echocardiography is the only real-time imaging modality used to monitor and guide RFA therapy [10,11]. Intracardiac ultrasound has been used to monitor ablation therapy in real time [12] by assessing RFA catheter tissue contact and contact angle[13], visualizing restenosis of pulmonary veins, and providing feedback for titration of RF energy to reduce incidence of embolic events due to over-treatment of cardiac tissue[14]. In particular, echocardiology is the standard imaging modality for real-time guidance of RFA of atrial fibrillation, to prevent adverse events to the esophagus[15].

Real-time monitoring and guidance can be further aided with spectroscopy to monitor lesion formation. This is especially important for complex cases such as treatment of atrial fibrillation and ventricular tachycardia. Previous studies have shown that the optical properties of heated myocardium (absorption, scattering and anisotropy coefficients) are significantly different from normal tissue[16]. Optical techniques such as OCT[17-19] and endoscopy[20] are being explored for RFA monitoring and guidance. NIRS can address unmet clinical needs of cardiac RFA therapy by assessing the contact and contact angle of the RF catheter with the tissue, confirming that a lesion has been formed when RF energy is delivered, detecting early damage, and measuring lesion depth.

Acute success and efficacy of ablation are determined through functional electrophysiology (EP) testing, to ensure that the lesions interrupt conduction. The ability to directly confirm that a lesion has been formed after energy delivery will eliminate ambiguity when EP testing shows that conduction interruption was not achieved, by eliminating the possibility the energy dose failed to result in a lesion. The ability to detect early damage could enable titration of energy delivery and reduce complication rates. Continuous wave diffuse reflectance spectroscopy (DRS) has been shown to provide useful insight in characterizing biological tissues[21, 22]. It is well known that backscattered reflectance changes can vary as a function of sample optical properties. Perturbations in specific regions of the visible and nearinfrared range may largely correspond to tissue absorption and scattering effects, which are understood to be closely related to tissue composition and particle size and density. We therefore hypothesize that DRS in the near-infrared region, or simply NIRS, can be used to characterize cardiac tissue changes during RFA therapy. We present preliminary results in the near-infrared spectroscopy-guided RFA in swine and canine hearts for tissue characterization. In addition, we extend the monitoring of spectral changes to the extraction of change in tissue absorption and reduced scattering using a diffusion-based model.

\section{MATERIALS AND METHODS}

\subsection{Near Infrared Spectroscopy (NIRS) System}

A near-infrared spectroscopy (NIRS) system was built in-house and data was acquired under two different configurations in this study. The first configuration employed an $840 \mathrm{~nm} \pm 40 \mathrm{~nm}$ LED as a light source and a spectrometer (Thorlabs, Newton, NJ, USA). Light was delivered onto the excised samples via a 400 micron optical fiber. Backscattered light was then routed to the spectrometer by a collection fiber fixed at approximately $1.5 \mathrm{~mm}$ from the illumination fiber. 
Tissue reflectance spectra acquired by this configuration was normalized to measurements obtained from a reflectance standard (Labsphere Inc.). A custom LabView program facilitated system synchronization and spectral data acquisition.

For the second configuration, a fiber optic catheter was designed to have a source-detector separation of 3.5 $\mathrm{mm}$. This separation was selected to allow for an inner channel for which the radiofrequency ablation catheter $(\sim 3 \mathrm{~mm}$ in diameter) could be routed into. In addition, the probe geometry enabled a more accurate sampling of the zone of resistive and conductive heating during ablation as compared to the $1.5 \mathrm{~mm}$; because of the small source-detector separation relative to the RF catheter tip, real-time measurements during tissue ablation for configuration 1 was limited to the periphery (lesion rim). Monte Carlo simulations were conducted for photons collected at this source-detector separation to better characterize the mean volume interrogated. A maximum depth of approximately 4-8 $\mathrm{mm}$ was determined for the range of optical properties relevant in biological tissues at near-infrared wavelengths. The LED light source was replaced with a Tungsten-Halogen light source in order to obtain diffuse reflectance data over a wider spectral range $(650-900 \mathrm{~nm})$.

\subsection{Tissue Preparation}

Fresh swine and canine hearts $(n=3)$ were acquired through Columbia University's Tissue Sharing Program. Hearts were sectioned for right and left atria and ventricular tissues and were supraperfused in phosphate buffered saline maintained at $37^{\circ} \mathrm{C}$. Optical measurements were taken on normal myocardium for each section with both optical configurations $(1.5 \mathrm{~mm}$ and $3.5 \mathrm{~mm})$. A total of 300 Spectra were acquired from normal myocardium, epicardium, epicardial fat, and the ablated myocardium using the $1.5 \mathrm{~mm}$ configuration. The spectra were then normalized to a Spectralon measurement and a wavelength dependent linear model was used to fit each spectra between 820-880 nm. Unless otherwise stated, for all ablation used in this study RF energy was then applied for $60 \mathrm{~s}$ at $30 \mathrm{~W}$. Spectra acquired during RFA were normalized to the baseline reading and a wavelength dependent linear model was used to fit each spectra between $820-880 \mathrm{~nm}$. The extracted slope values were compared across the hearts for $1.5 \mathrm{~mm}$ configuration. Dynamic changes in slope during RF ablation were compared for both configurations.

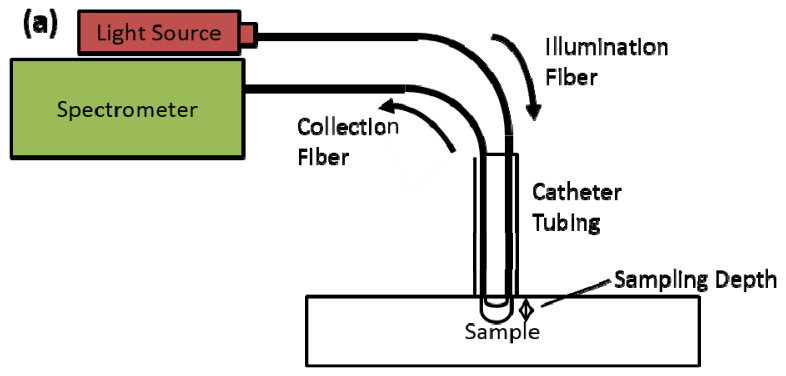

(b)

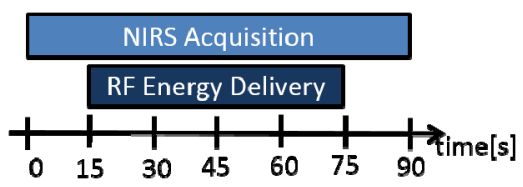

(c)

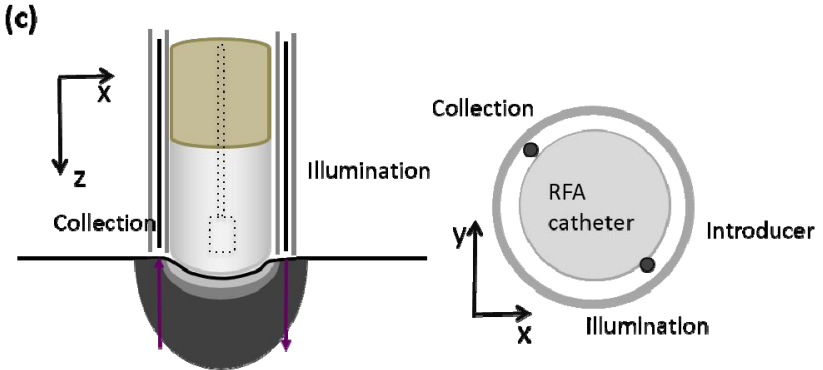

Figure 1. (a) NIRS system schematic diagram. b) shows the sequence of data acquisition for time course reflectance measurements. (c) Dynamic changes in diffuse reflectance are monitored from the zone of resistive and conductive heating during radiofrequency ablation for the $3.5 \mathrm{~mm} \mathrm{S-D}$ configuration and (d) along the tissue-catheter periphery for the $1.5 \mathrm{~mm}$ configuration. 


\subsection{Spectral Analysis}

Reflectance spectra acquired with the $1.5 \mathrm{~mm}$ configuration were first normalized to measurements obtained from a diffuse reflectance standard. The resulting spectra obtained from different myocardial tissue were observed to be relatively linear within the range of 800-880 nm Therefore, the normalized spectra, $R_{\text {norm }}$, were fitted to a wavelengthdependent linear model as described below

$$
R_{\operatorname{markx}}=a \dot{\alpha}+b
$$

where $\lambda$ is the wavelength in nanometers and $a$ and $b$ is the slope and intercept values, respectively. Extracted slopes were compared were then compared across tissues and during RFA treatment.

A forward model was developed similar to Palmer et. al. to describe diffuse reflectance as a function of optical coefficients of absorption and reduced scattering for the $3.5 \mathrm{~mm}$ configuration [23]. In contrast however, a diffusion theory-based model was employed instead of a precalculated, Monte Carlo-based lookup table. Absorption was modeled as a linear combination of oxygenated myoglobin, de-oxygenated myoglobin, metmyoglobin, lipid, and water molar extinction spectra. Scattering was modelled by Mie Theory for a given scatterer density and mean scatterer radius. In order to measure the change in optical properties between normal and ablated endocardium, reflectance from the ablated endocardium was normalized to the pre-ablation spectra. Finally, a least squares minimization was used to fit the normalized spectra to the diffusion-based forward model, keeping chromophore concentration, scatterer density, and mean scatterer radius as free parameters.

\section{RESULTS AND DISCUSSION}

We prototyped a NIRS system, which allowed for characterizing cardiac tissue optical properties dynamically with the application of radiofrequency energy. Our system was tested ex vivo within swine and canine hearts within 48 hours after death.

Reflectance spectra were acquired from four different types of swine cardiac tissue normal endocardium, ablated endocardium, normal epicardium, and epicardial fat. Calibrated spectra were fitted to a wavelength-dependent linear model (800-880nm), and slope values were extracted for comparison. Slope values for normal endocardium were approximately 2-3 fold less than that of the ablated values, Figure 2(a). Epicardial fat slopes were on the order of 1.5 to 2 fold greater than the normal epicardium values. This suggests that without any history, a simple threshold classifier can potentially be used to distinguish these types of cardiac tissue.

(a)

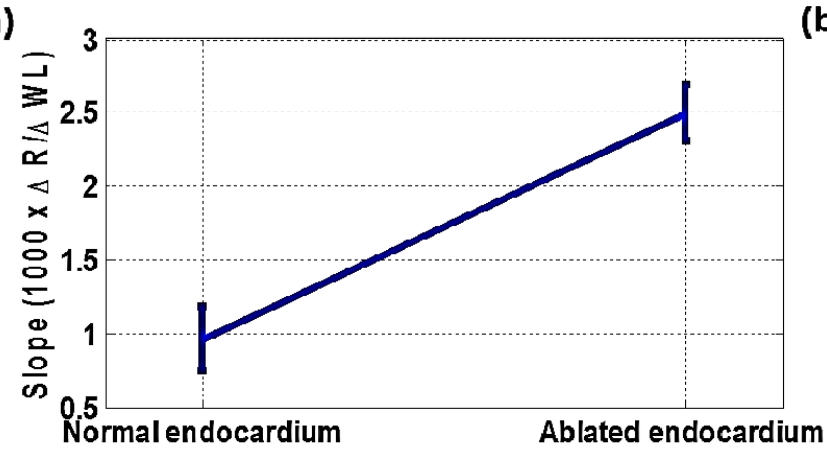

(b)

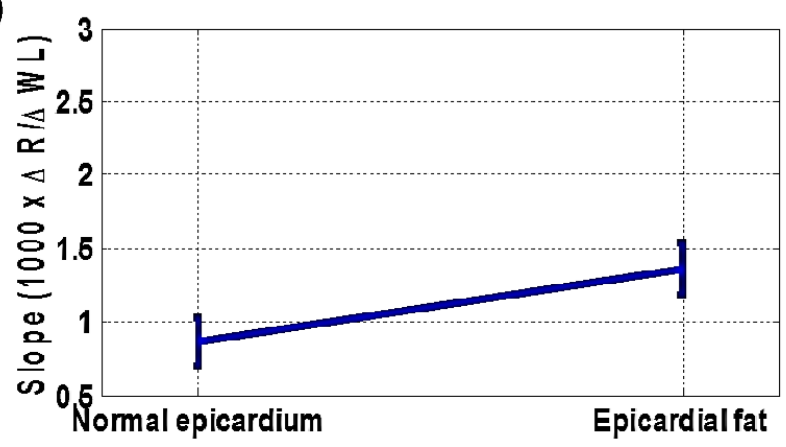

Figure 2. Mean and standard deviations from extracted reflectance slope values for the $1.5 \mathrm{~mm}$ configuration are shown for relevant tissue regions. (a) compares normal $(0.98 \pm 0.22)$ from ablated endocardium $(2.49 \pm 0.19)$ and (b) compares of normal epicardium $(0.86 \pm 0.15)$ to epicardial fat. $(1.3 \pm 0.12)(n=300)$ 
Slope changes were monitored during radiofrequency ablation at the zone of resistive and conductive heating $(3.5 \mathrm{~mm}$ configuration) and along the lesion rim (1.5 mm configuration), Figure 3. Values obtain from the rim show a rise from the onset of treatment and is sustained throughout the time course of the ablation. In contrast, a sharp rise was seen in slopes obtained from within the direct lesion site followed by an immediate plateauing effect.
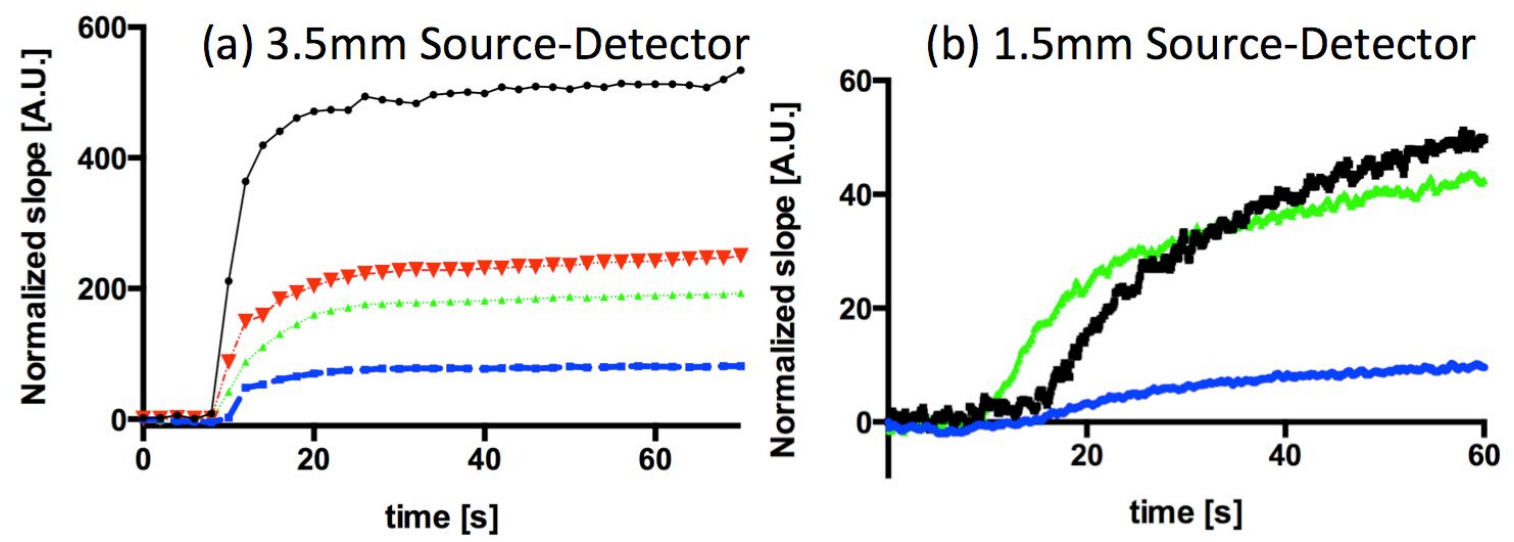

Figure 3. Dynamic changes in slope of reflectance spectra with the application of RF energy for varying catheter configurations. (a) $3.5 \mathrm{~mm}$ source and detector fiber distance resulted in plateau of slope versus time plot for various lesions. (b) $1.5 \mathrm{~mm}$ source and detector fiber distance resulted in near linear change in slope versus time for various lesions.

Due to the wider spectral range of the $3.5 \mathrm{~mm}$ configuration system $(650-900 \mathrm{~nm})$, a multi-parametric fit was employed which included the known extinction spectra for oxymyoglobin, deoxymyglobin, metmyoglobin, lipid, and water, in addition to a scattering model based on Mie Theory. A diffusion theory-based forward model fitted to the final reflectance spectra post-ablation to determine the net change in scattering and absorption (Figure 4)[23]. It was found that majority of absorption changes were induced by an increase in tissue metmyoglobin content. In addition, an increase in reduced scattering was observed primarily caused by an increase in slope. The real-time extraction of optical properties, as shown in Fig. 4, can provide the clinician with valuable insight into the extent of lesion generation along with tissue composition.
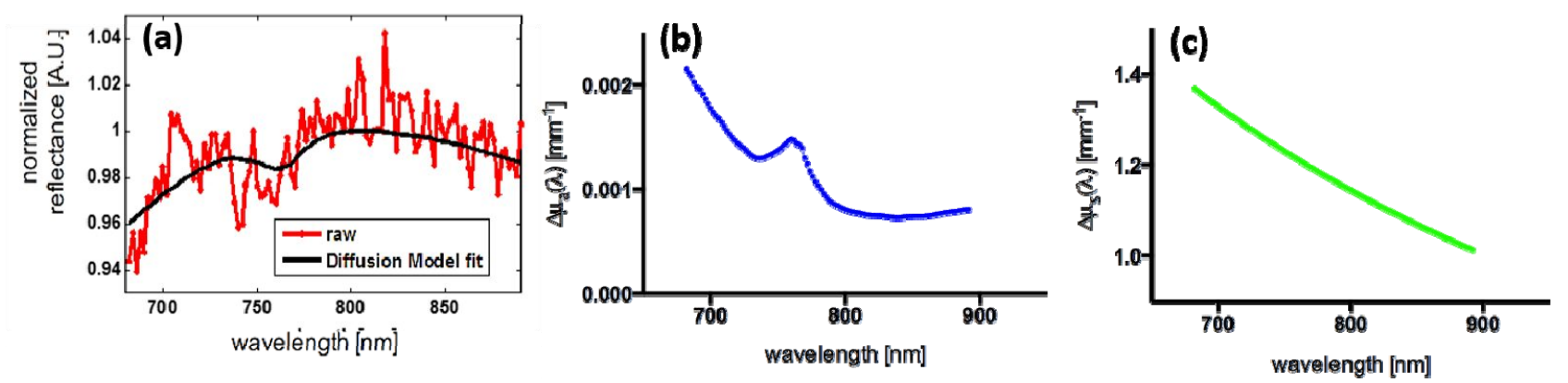

Figure 4. Extracting absorption and scattering spectra from normalized reflectance spectra from right atrium myocardium, postablation. Treated tissue reflectance spectra was normalized to the pre-ablated, baseline spectra and fitted to the geometry-specific, diffusion theory-based reflectance model. (a) shows the normalized raw data (red) along with the model fit superimposed (black). (b) shows the extracted absorption and (c) reduced scattering spectra. 
It should be noted that ex vivo data may not be completely reflective of changes that occur in vivo tissue for a number of reasons. Increased hemoglobin content, for instance, could increase effective tissue absorption, leading to a reduction in maximum depth of the interrogation as seen from Monte Carlo simulations. However, concentrations of red blood cells in the myocardium in vivo have been previously reported to be between 2-9 \% [16]. Nonetheless, future studies will investigate the hemoglobin influence on the metrics obtained for tissue classification. In addition, future studies will be aimed at determining an empirical relationship between tissue optical properties and lesion characteristics. Fresh tissue stained with TTC could expose the extent of RFA induced necrosis thereby revealing lesion depth, a crucial parameter in determining effective lesion generation.

\section{CONCLUSION}

In conclusion, we present metrics for discriminating cardiac tissues and monitoring dynamic changes in tissue optical properties during RFA using NIRS. Measurements obtained Results from this study encourage the application NIRS tools for the assessment of myocardium during treatment.

\section{Acknowledgement}

The authors would like to thank Yang Zhao for help in operating the radiofrequency ablation equipment and Columbia University's Institute of Comparative Medicine for providing the cardiac tissues used in this study. The authors will also like to thank our funding sources: Columbia University Provost Small Grants, NIH Loan Repayment Program and NSF BRIGE Grant EEC-1342273.

\section{REFERENCES}

1. Rosamond, W., K. Flegal, K. Furie, A. Go, K. Greenlund, N. Haase, S.M. Hailpern, M. Ho, V. Howard, B. Kissela, S. Kittner, D. Lloyd-Jones, M. McDermott, J. Meigs, C. Moy, G. Nicho, C. O’Donnell, V. Roger, P. Sorlie, J. Steinberger, T. Thom, M. Wilson, and Y. Hong, Heart Disease and Stroke Statistics 2008 Update: A Report From the American Heart Association Statistics Committee and Stroke Statistics Subcommittee. Circulation, 2008. 117: p. e25-e146.

2. Huang, S.K.S. and M.A. Wood, Catheter Ablation of Cardiac Arrhythmias. 2006: Saunders.

3. Marrouche, N., J. Guenther, N. Segerson, M. Daccarett, H. Rittger, H. Marschang, V. Schibgilla, M. Schmidt, G. Ritscher, G. Noelker, and J. Brachmann, Randomized comparison between open irrigation technology and intracardiac-echo-guided energy delivery for pulmonary vein antrum isolation: procedural parameters, outcomes, and the effect on esophageal injury. J Cardiovasc Electrophysiol. , 2007. 18(6): p. 583-8.

4. Brockman, R., Cardiac Ablation Catheters Generic Arrhythmia Indications for Use; Guidance for Industry, 2002, FDA Center for Devices and Radiological Health. Cardiac Electrophysiology and Monitoring Branch Division of Cardiovascular and Respiratory Devices Office of Device Evaluation: Rockville, MD.

5. Cappato, R., H. Calkins, S.-A. Chen, W. Davies, Y. Iesaka, J. Kalman, Y.-H. Kim, G. Klein, D. Packer, and A. Skanes, Worldwide Survey on the Methods, Efficacy, and Safety of Catheter Ablation for Human Atrial Fibrillation. Circulation, 2005. 111: p. 1100-1105.

6. Dickfeld, T., R. Kato, M. Zviman, S. Nazarian, H. Ashikaga, A.C. Lardo, R.D. Berger, H. Calkins, and H. Halperin, Characterization of Acute and Subacute Radiofrequency Ablation Lesions with Non-enhanced Magnetic Resonance Imaging. Heart Rhythm, 2007. 4(2): p. 208-14.

7. Dickfeld, T., R. Kato, M. Zviman, S. Lai, G. Meininger, A.C. Lardo, A. Roguin, D. Blumke, R. Berger, H. Calkins, and H. Halperin, Characterization of Radiofrequency Ablation Lesions with Gadolinium-Enhanced Cardiovascular Magnetic Resonance Imaging. J Am Coll Cardiol, 2006. 47(2): p. 370-8.

8. Abbara, S., J.C. Desai, R.C. Cury, J. Butler, K. Nieman, and V. Reddy, Mapping epicardial fat with multidetector computed tomography to facilitate percutaneous transepicardial arrhythmia ablation. European Journal of Radiology, 2006. 57(3): p. 417-422. 
9. Sarin, S., C. Wenger, A. Marwaha, A. Qureshi, B.D. Go, C.A. Woomert, K. Clark, L.A. Nassef, and J. Shirani, Clinical significance of epicardial fat measured using cardiac multislice computed tomography. American Journal of Cardiology, 2008. 102(6): p. 767-771.

10. Alaeddini, J., M.A. Wood, B.P. Lee, and K.A. Ellenbogen, Incidence, Time Course, and Characteristics of Microbubble Formation During Radiofrequency Ablation of Pulmonary Veins with an 8-mm Ablation Catheter. Pace, 2006. 29: p. 979-984.

11. David Schwartzman, M., R. John Nosbisch, and R. Debra Housel, Echocardiographically guided left atrial ablation: Characterization of a new technique. Heart Rhythm Society, 2006. 3(8): p. 930-938.

12. Citro, R., V. Ducceschi, A. Salustri, M. Santoro, M. Salierno, and G. Gregorio, Intracardiac echocardiography to guide transseptal catheterization for radiofrequency catheter ablation of left-sided accessory pathways: two case reports. Cardiovascular Ultrasound 2004. 2(20).

13. Ren, J.-F. and F.E. Marchinski, Utility of Intracardiac Echocardiography in Left Heart Ablation for Tachyarrhythmias. Echocardiography, 2007. 24: p. 533-540.

14. Wright, M., E. Harks, S. Deladi, F. Suijver, Maya Barley, A.v. Dusschoten, S. Fokkenrood, P. Fei Zuo, F. Sacher, M. Hocini, M. Haïssaguerre, and P. Jaïs, Real-time lesion assessment using a novel combined ultrasound and radiofrequency ablation catheter. Heart Rhythm, 2011. 8(2): p. 304-12.

15. Ren, J.-F., M. David Lin, F.E. Marchlinski, D.J. Callans, and V. Patel, Esophageal imaging and strategies for avoiding injury during left atrial ablation for atrial fibrillation. Heart Rhythm, 2006. 3(10): p. 1156-1161.

16. Swartling, J., S. Palsson, P. Platonov, S.B. Olsson, and S. Andersson-Engels, Changes in tissue optical properties due to radio-frequency ablation of myocardium. Med Biol Eng Comput, 2003. 41: p. 403-9.

17. Fleming, C.P., K.J. Quan, and A.M. Rollins, Toward guidance of epicardial cardiac radiofrequency ablation therapy using optical coherence tomography. Journal of Biomedical Optics, 2010. 15(4): p. 041510.

18. Fleming, C.P., K.J. Quan, H. Wang, G. Amit, and A.M. Rollins, In vitro characterization of cardiac radiofrequency ablation lesions using optical coherence tomography. Optics Express, 2010. 18(3): p. 30793092.

19. Fleming, C.P., N. Rosenthal, A.M. Rollins, and M. Arruda, First in vivo Real-- Time Imaging of Endocardial Radiofrequency Ablation by Optical Coherence Tomography: Implications on Safety and The Birth of 'Electro- structural" Substrate-- Guided Ablation. Innovations in Cardiac Rhythm Management, 2011. 2: p. $199-201$.

20. Wissner, E., A. Metzner, B. Reissmann, P. Rausch, A. Bardyszewski, C. Lemes, R. Tilz, A. Rillig, S. Mathew, S. Deiss, F. Ouyang, and K. Kuck, Wide Circumferential versus Individual Isolation of Pulmonary Veins Using the Endoscopic Ablation System. J Cardiovasc Electrophysiol, 2013. Epub ahead of print.

21. Reif, R., M.S. Amorosino, K.W. Calabro, O. A'Amar, S.K. Singh, and I.J. Bigio, Analysis of changes in reflectance measurements on biological tissues subjected to different probe pressures. J Biomed Opt, 2008. 13(1): p. 010502.

22. Mourant, J.R., T.M. Johnson, G. Los, and I.J. Bigio, Non-invasive measurement of chemotherapy drug concentrations in tissue: preliminary demonstrations of in vivo measurements. Physics in Medicine and Biology, 1999. 44(5): p. 1397-1417.

23. Palmer, G.M. and N. Ramanujam, Monte Carlo-based inverse model for calculating tissue optical properties. Part I: Theory and validation on synthetic phantoms. Applied Optics, 2006. 45(5): p. 1062-1071. 